\title{
Los debates electorales, ¿el último reducto frente la mediatización? Un estudio de caso de las elecciones generales españolas de $2015^{1}$
}

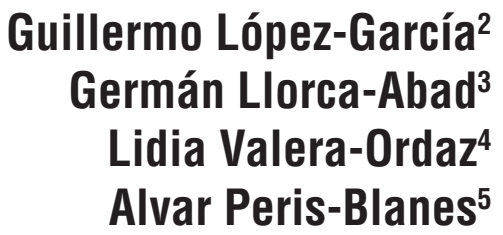

Recibido: 2017-05-05

Enviado a pares: $2017-05-15$
Aprobado por pares: 2017-06-01

Aceptado: 2017-07-13

DOI: $10.5294 /$ pacla.2018.21.3.6

Para citar este artículo / to reference this article / para citar este artigo López-García, G., Llorca-Abad, G., Valera-Ordaz, L. y Peris-Blanes, A. (2018). Los debates electorales, ¿el último reducto frente la mediatización? Un estudio de caso de las elecciones generales españolas de 2015. Palabra Clave, 21(3), 772-797. DOI: 10.5294/ pacla.2018.21.3.6

\section{Resumen}

La mediatización de la política alcanza su máxima expresión con motivo de las campañas electorales, en las que los actores políticos se afanan por lanzar sus mensajes persuasivos al espacio público, con el fin de acceder $y$, en última instancia, convencer al electorado. Este artículo se propone analizar el discurso político en los debates electorales televisados durante la campaña de las elecciones generales españolas de diciembre de 2015. El

1 Este trabajo forma parte del proyecto de investigación Mediaflows (www.mediaflows.es, CSO2016-77331-C2-1-R), financiado por el Ministerio de Economía y Competitividad de España.

2 orcid.org/0000-0002-5701-2024. Universitat de València, España. guillermo.lopez@uv.es

3 orcid.org/0000-0002-0934-8420. Universitat de València, España. german.llorca@uv.es

4 orcid.org/0000-0002-1085-980X. Universitat de València, España. lidia.valera@uv.es

5 orcid.org/0000-0002-2323-2766. Universitat de València, España. alvar.peris@uv.es 
objetivo del análisis es observar qué temas destacaron en las intervenciones de los actores políticos y determinar si la mediatización afecta el contenido de los debates electorales, o si, por el contrario, estos espacios se mantienen como foros para la discusión detallada de diversas cuestiones de política pública. Para ello, se propone un análisis de contenido sustanciado en setenta categorías de análisis, agrupadas en cuatro bloques: policy issues, political issues, campaign issues y personal issues. Los resultados indican claramente que las cuestiones de política sectorial dominan el discurso político, de forma que los debates electorales, pese al alcance de la mediatización, constituyen todavía espacios para la exposición detallada y la confrontación de políticas públicas.

\section{Palabras clave}

Elecciones, campaña electoral, debates, comunicación política, mediatización, televisión (Fuente: Tesauro de la Unesco). 


\section{Electoral Debates. The Last Stronghold Against Mediatization? A Case Study of the 2015 Spanish General Elections}

\section{Abstract}

The Mediatization of politics reaches its maximum expression with electoral campaigns, in which political actors strive to launch their persuasive messages to the public, in order to reach and, ultimately, convince the voters. This article aims to analyze the political discourse in the electoral debates televised during the 2015 Spanish Presidential Elections. The objective of the analysis is to observe which topics stood out during the political actors' interventions and to determine if mediatization affects the content of the electoral debates, or if, on the contrary, these spaces remain as forums for the detailed discussion of various public policy issues. For this purpose, we propose an analysis of content based on seventy categories of analysis, grouped in four blocks: policy issues, political issues, campaign issues and personal issues. The results clearly indicate that sectoral policy matters control the political discourse; therefore, electoral debates, despite the reach of mediatization, still constitute spaces for detailed exposition and the confrontation of public policies.

\section{Keywords}

Elections; electoral campaign; debates; political communication; mediatization; television (Source: Unesco Thesaurus). 


\section{Os debates eleitorais: 0 último reduto frente à midiatização? Um estudo de caso das eleições gerais espanholas de 2015}

\section{Resumo}

A midiatização da política apresenta sua máxima expressão nas campanhas eleitorais, nas quais os atores políticos se esforçam para lançar suas mensagens persuasivas no espaço público com a finalidade de alcançar e, em última instância, convencer o eleitorado. Este artigo se propõe a analisar o discurso político nos debates eleitorais televisados durante a campanha das eleições gerais espanholas de dezembro de 2015 . O objetivo da análise é observar quais temas tiveram destaque nas intervenções dos atores políticos e determinar se a midiatização afeta o conteúdo dos debates eleitorais, ou se, pelo contrário, esses espaços se mantêm como fóruns para a discussão detalhada de diversas questões de política pública. Para isso, propõe-se uma análise de conteúdo baseada em setenta categorias de análises, organizadas em quatro blocos: policy issues, political issues, campaign issues e personal issues. Os resultados indicam claramente que as questões de política setorial dominam o discurso político, de forma que os debates eleitorais, apesar do alcance da midiatização, ainda constituem espaços para a exposição detalhada e para o confronto de políticas públicas.

\section{Palavras-chave}

Campanha eleitoral; comunicação política; debates; eleições; midiatização; televisão (Fonte: Tesauro da Unesco). 


\section{Introducción: debates televisados y mediatización de la política}

Durante la campaña de las elecciones generales españolas del 20 de diciembre de 2015, las cuestiones relacionadas con los debates concitaron una enorme atención, en parte como resultado de la particular coyuntura política, caracterizada por la irrupción de dos nuevos partidos políticos en el sistema político español (Podemos y Ciudadanos), y en parte como resultado de la enorme presencia de los candidatos de los nuevos partidos en distintos formatos televisivos. Hasta este momento, en España solo se habían celebrado cinco debates electorales entre candidatos en televisión: dos entre Felipe González (Partido Socialista Obrero Español [PSOE]) y José María Aznar (Partido Popular [PP]) en 1993, dos entre el socialista José Luis Rodríguez Zapatero y el conservador Mariano Rajoy en 2008, y un debate entre Alfredo Pérez Rubalcaba y Mariano Rajoy en 2011.

No obstante, el electorado español está habituado a la presencia de los actores políticos en televisión, y puede decirse que los telespectadores españoles han naturalizado con extraordinaria rapidez la existencia de debates televisados entre los principales candidatos que concurren a unas elecciones, dado el modelo de personalización de la política que propicia el medio televisivo (Rodríguez, Jandura y Rebolledo, 2014, p. 63). Aunque los debates electorales considerados importantes en España son, sobre todo, los protagonizados por los cabezas de lista de cada partido, no hay que desdeñar la existencia de aquellos en los que participan otros actores políticos. Solo hay que recordar la expectación levantada en su momento por el debate entre Manuel Pizarro (PP) y Pedro Solbes (PSOE) en 2008, en pleno fragor de la crisis financiera, o el debate "Primero las mujeres" (así fue presentado por la televisión encargada de organizarlo, Antena $3 \mathrm{TV}$ ), protagonizado por Andrea Levy (PP), Margarita Robles (PSOE), Carolina Bescansa (Podemos) e Inés Arrimadas (Ciudadanos) en 2016.

Sin embargo, las diferencias que existen entre aquellos primeros debates y los actuales son sustanciales, sobre todo a tenor de cómo ha cambiado el contexto en que ambos se produjeron. Conviene destacar tres grandes transformaciones. En primer lugar, la multiplicación de agentes emisores, 
dada la transformación del contexto tecnológico y comunicativo, que incluye tanto la proliferación de canales digitales de televisión como la de numerosas plataformas de internet con gran potencial de difusión (Bennett e Iyengar, 2008). En segundo lugar, el aumento del número de partidos políticos con opciones de alcanzar la posibilidad de formar o entrar en el Gobierno, como ya adelantábamos, con la irrupción de fuerzas políticas como Podemos y Ciudadanos. En efecto, el sistema político español ha experimentado recientemente una transformación de un sistema de partidos básicamente bipartidista (aunque con la presencia de algunas fuerzas políticas minoritarias con un fuerte apoyo localizado, como los partidos nacionalistas catalanes y vascos, y con otros partidos con un apoyo electoral disperso y con menos capacidad para alcanzar representación parlamentaria, como Izquierda Unida), en el que únicamente el PSOE y el PP tenían posibilidades reales de gobernar, a un sistema tetrapartidista, en el que son cuatro las formaciones políticas que se reparten el grueso de la representación parlamentaria, y con ella, la posibilidad de formar gobierno. Y, en tercer lugar, conviene destacar también la diversificación de formatos televisivos en los que presentar la confrontación dialéctica de los candidatos políticos, es decir, la evolución de la presentación de los debates electorales hacia una multiplicidad de formatos audiovisuales, que incluyen diversas fórmulas de infoentretenimiento.

Asimismo, las características particulares de la televisión, en cuanto medio con gran influencia social y que facilita la exposición mediática transversal de los individuos (Goldman y Mutz, 2011), es decir, la exposición a mensajes que no han elegido antes y que no necesariamente concuerdan con sus convicciones ideológicas, hacen de los debates electorales televisados una ocasión persuasiva particularmente propicia para los partidos. En los debates electorales, en definitiva, los candidatos tienen la oportunidad de difundir sus mensajes persuasivos entre públicos heterogéneos, de acceder a telespectadores a los que generalmente no podrían alcanzar merced a la tendencia a la exposición selectiva, es decir, la preferencia de exposición a contenidos que concuerdan con las ideas y los valores preexistentes de la audiencia (Humanes, 2014, p. 780). 
Abundando en esta perspectiva, y según indica García Luengo (2011), los debates electorales aportan importantes especificidades a la campaña electoral: 1) permiten a los ciudadanos escuchar directamente a los candidatos sin intermediación mediática, 2) generan audiencias elevadas, con lo que llegan a electores a los que normalmente no alcanza la información política y 3) concentran la atención de la campaña y generan mucha información añadida. Los debates televisados, además, pueden tener efectos significativos sobre la decisión del voto, contribuyendo a la formación de las preferencias electorales de los indecisos (Barreiro, Pereira y García, 2015, p. 74). Asimismo, diversos autores destacan el papel central del medio televisivo como medio de referencia a través del cual se informa la mayoría de los votantes frente a otras formas de comunicación (Lago y Martínez, 2013; Rodríguez y Bouza, 2008).

Desde un punto de vista histórico, los debates televisados celebrados en anteriores comicios fueron grandes éxitos de audiencia, "con ratings comparables tan solo a los grandes eventos deportivos (superiores al $60 \%$ de media)" (García, 2015, p. 137). En esta línea, conviene destacar que en los últimos años se ha producido un significativo incremento del papel de la televisión como vehículo privilegiado de escenificación de alternativas políticas, conflictos, estrategias y debates de toda clase. La aparición en la escena política de los ya mencionados partidos "emergentes" (Podemos y Ciudadanos), la desafección de muchos ciudadanos respecto de la política tradicional y el afán por encontrar nuevos espacios y formatos políticos son todos ellos fenómenos con los que la televisión se halla asociada de manera indisoluble. Cabría considerar, incluso, que la televisión ha condicionado la naturaleza de los mensajes políticos y de los liderazgos de las principales opciones electorales (Blumler y Gurevitch, 1995; Mazzoleni, 2010).

Los medios de comunicación, especialmente la televisión, tienen una gran capacidad para establecer los problemas que preocupan a la ciudadanía, tal y como han demostrado sucesivos estudios de agenda setting (McCombs y Shaw, 1972; Rodríguez y Bouza, 2008; Iyengar y Kinder, 2010). Asimismo, la aparición de nuevos formatos televisivos que intentan adecuar la lógica de la discusión política a las preferencias de la audiencia y los programadores 
de televisión, con proliferación de tertulias y programas de infoentretenimiento político, también afectó considerablemente la naturaleza y estructuración de algunos de los debates de la campaña de 2015.

En el contexto de las elecciones que nos ocupan, los debates electorales adquirieron mayor relevancia, continuidad y dimensión merced a la complementariedad entre su escenificación en televisión y los formatos televisivos creados específicamente para comentar, resumir y caracterizar su desarrollo, organizados por los propios medios de comunicación (el "debate del debate" en prensa, radio y televisión). Pero el impacto de los debates en la esfera pública fue particularmente acusado merced a la reverberación de los contenidos mediáticos por medio de la acción de comentaristas políticos, líderes de opinión y ciudadanos anónimos en las redes sociales.

Es decir, la combinación de diversos dispositivos y emisores en el contexto de un espacio público expandido merced a la combinación de medios tradicionales y digitales incrementa notablemente la relevancia de los debates electorales. No solo llegan a la audiencia que los sigue en directo, sino también a otros segmentos de la población gracias a que son comentados a través de los medios digitales, redifundidos por los medios tradicionales mediante resúmenes de los momentos más significativos, y comentados mediante toda clase de fuentes secundarias. Los debates electorales, en suma, son un excelente ejemplo del funcionamiento del sistema híbrido de comunicación postulado por Chadwick (2013), en virtud del cual los viejos y nuevos medios se combinan, asocian y replican entre sí en relación con el tratamiento informativo de cualquier acontecimiento, y los públicos realizan un consumo simultáneo y combinado de múltiples plataformas comunicativas (Vaccari, Chadwick y O'Loughlin, 2015; Vergeer y Franses, 2016; Ceron y Splendore, 2016).

En definitiva, los debates electorales, desde el punto de vista de su incidencia en la agenda pública, constituyen un caso digno de estudio específico, pues generan una ventana de oportunidad para que los actores políticos puedan trasladar sus mensajes directamente a la audiencia, sin apenas intermediación periodística. Especialmente en el caso de países como España, 
donde a menudo la labor de los moderadores de los debates electorales se ha limitado a asignar los turnos de palabra y controlar los tiempos. Pero, al mismo tiempo, han de hacerlo teniendo muy presente la repercusión no solo entre el público que está siguiendo el debate en directo, sino también entre los que lo comentan en las redes sociales (Vergeer y Franses, 2016) y en el "posdebate" orquestado por los medios, que se encargan de dictaminar quién ha ganado el debate, con los importantes efectos electorales que esto podría conllevar (Schrott, 1990), y cuáles han sido las temáticas y los momentos más importantes de este; que, además, se plantean en un escenario caracterizado por la diversificación de medios, enfoques y formatos.

En otras palabras, los debates electorales constituyen un campo de juego privilegiado de disputa entre actores políticos que intentan influir en la construcción de la agenda mediática y, en última instancia, dar forma a las preocupaciones de los ciudadanos, en un contexto - que rara vez va a repetirse - de grandes audiencias e intensificación del interés por consumir contenidos políticos (Zaller, 1992).

\section{Metodología}

\section{Preguntas de investigación}

Este estudio aspira a investigar el discurso de los actores políticos durante los debates electorales, con el fin de observar si tales espacios constituyen foros para la exposición pormenorizada y el debate de distintos enfoques de política sectorial, como en teoría propiciaría el formato de estos encuentros, en los que se plantean preguntas y áreas temáticas de interés a los candidatos, o si, por el contrario, estos están dominados por las tendencias atribuibles a la mediatización de la política (Schulz, 2004; Strömbäck, 2008), es decir, por centrarse, entre otros, en cuestiones relativas a las estrategias de campaña, y en asuntos relacionados con la personalidad de los candidatos; cuestiones, en definitiva, fomentadas por un enfoque mediático espectacularizado e inscrito en la lógica del infoentretenimiento, cuyo objetivo es obtener grandes audiencias. En otras palabras, el estudio se propone responder a la siguiente pregunta de investigación: 
P1: ¿Qué tipo de cuestiones prevalecen en el discurso de los actores políticos durante los debates electorales?

\section{Diseño metodológico}

Con el fin de responder a esta pregunta de investigación, se propone una aproximación metodológica cuantitativa, sustanciada en una técnica de investigación específica: el análisis de contenido. Conviene señalar que el diseño metodológico específico, compuesto por una serie de categorías de análisis temático que dan cuenta de qué cuestiones dominan el discurso de los actores políticos, ha sido previamente utilizado y validado en diversos trabajos desarrollados en el grupo de investigación al que los autores pertenecen (López-García et al., 2015; López-García y Valera-Ordaz, 2017).

El análisis se centra, por tanto, en el contenido manifiesto de los mensajes emitidos por los actores políticos durante los debates electorales. Estos mensajes constituyen una puerta de entrada al discurso y la agenda programática de los líderes políticos, es decir, a sus principales preocupaciones de política sectorial y estrategias de campaña. Analizar pormenorizadamente el contenido de sus intervenciones televisivas durante los debates nos permite identificar qué tipo de discurso prevalece en estos espacios y detectar las claves temáticas de sus mensajes, y establecer posibles comparaciones entre las agendas de los distintos actores políticos; es decir, el diseño metodológico se basa en un análisis temático. La identificación de los temas se ha realizado por medio de setenta categorías de análisis que hemos clasificado en cuatro bloques (policy, campaign, political issues y personal issues), siguiendo la tipología establecida por Patterson (1980), citado por Mazzoleni (2010). ${ }^{6}$ Más concretamente:

Political issues: cuestiones concernientes a la esfera más abstracta de la confrontación electoral, ideología de los partidos y candidatos, alianzas entre partidos, relaciones con la sociedad civil y los poderes fácticos.

Policy issues: cuestiones de política sectorial, como la sanidad, la educación, la economía, etc.

6 Puede consultarse el listado específico de categorías de análisis para los temas en el anexo 1. 
Personal issues: cuestiones relativas a la vida y la actividad de los candidatos, su carácter, sus aficiones, etc.

Campaign issues: cuestiones relacionadas con el devenir de la campaña electoral, como actos de campaña específicos, sondeos, configuración de listas y candidaturas, etc.

El corpus seleccionado fue analizado por dos codificadores, convenientemente entrenados para analizar las variables propuestas y aplicar los mismos criterios en la codificación mediante un libro de códigos diseñado al efecto. Con el fin de garantizar la fiabilidad de la codificación, se realizó un test de fiabilidad entre los dos codificadores, efectuado tras consensuar la segmentación por bloques de los treinta ítems designados. Concretamente, la prueba de fiabilidad seleccionada fue el porcentaje de acuerdo, que se considera un indicador de fiabilidad suficiente. Los resultados del test de fiabilidad señalan un acuerdo lo suficientemente alto entre codificadores: $96 \%$ de porcentaje de acuerdo en lo que se refiere a los cuatro bloques temáticos principales y $83 \%$ de porcentaje de acuerdo en el caso de las categorías específicas de análisis temático.

\section{La muestra}

Respecto de la selección de la muestra, se ha optado por analizar el contenido de cuatro encuentros televisivos: 1) el debate organizado por Atresmedia el 7 de diciembre, 2) el encuentro visto en TVE1, Canal 24 Horas, TVE Internacional y RTVE.es el 9 de diciembre, 3 ) el cara a cara entre Mariano Rajoy (PP) y Pedro Sánchez (PSOE) de La Academia de la TV emitido el 14 de diciembre y 4) el programa de Jordi Évole, Salvados, del 18 de octubre, protagonizado por Albert Rivera (Ciudadanos) y Pablo Iglesias (Podemos). Estos cuatro encuentros fueron, sin lugar a dudas, los debates más representativos de todos cuantos se celebraron, tanto por las cifras de audiencia como por su repercusión en redes sociales. El principal criterio de selección de estos formatos nos remite a su carácter estrictamente televisivo, lo cual nos permitió descartar otro tipo de encuentros, como aquellos organizados por algunos diarios o emisoras de radio.

El corpus de análisis queda, por tanto, circunscrito a estos cuatro formatos televisivos. De todos ellos, solo el programa de La Sexta, Salvados, 
incumple parcialmente las características formales de un debate televisivo tradicional, por varios motivos. En primer lugar, por la falta de emisión en directo, algo que se desvía significativamente de la lógica de los debates electorales tradicionales, en los que los candidatos discuten todo tipo de cuestiones en tiempo real. En segundo lugar, porque se trata de un encuentro editado por Salvados, lo cual también supone una notable diferencia con respecto a los debates electorales tradicionales, en los que no concurre una labor de edición a posteriori por parte del programa, sino que son emitidos en directo. En tercer lugar, porque se produjo fuera del escenario tradicional del estudio televisivo, y tuvo lugar en ambiente poco habitual: una cafetería barcelonesa. Pese a estas diferencias, este encuentro concitó una gran atención y tuvo un gran impacto en el debate público, por lo cual entendemos que reviste interés suficiente para ser incluido en el estudio. En la tabla 1 , se recopila la información básica sobre los debates electorales seleccionados para el análisis, que incluye el nombre del encuentro, la cadena de televisión o grupo de comunicación que organizó el encuentro, la fecha del debate, la duración del encuentro y la identidad del moderador. En total, se han analizado seis horas y media de emisión (390 min).

Tabla 1. Resumen de los debates electorales

\begin{tabular}{|c|c|c|c|c|}
\hline Debate electoral & $\begin{array}{c}\text { Cadena de } \\
\text { televisión/grupo de } \\
\text { comunicación }\end{array}$ & Fecha del debate & $\begin{array}{c}\text { Duración del } \\
\text { encuentro }\end{array}$ & Moderador \\
\hline El debate a 4 de Atresmedia & $\begin{array}{c}\text { Atresmedia } \\
\text { (Antena 3 y La Sexta) }\end{array}$ & $\begin{array}{c}7 \text { de diciembre } \\
\text { de } 2015\end{array}$ & 120 min & $\begin{array}{c}\text { Vicente Vallés } \\
\text { Ana Pastor }\end{array}$ \\
\hline El debate más plural & $\begin{array}{c}\text { Televisión Española } \\
\text { (TVE1) }\end{array}$ & $\begin{array}{c}9 \text { de diciembre } \\
\text { de } 2015\end{array}$ & 90 & Julio Somoano \\
\hline El cara a cara & $\begin{array}{c}\text { La Academia de } \\
\text { Televisión }\end{array}$ & $\begin{array}{c}14 \text { de diciembre } \\
\text { de } 2015\end{array}$ & Manuel Campo Vidal \\
\hline El debate de Salvados & La Sexta (Salvados) & $\begin{array}{c}18 \text { de Octubre } \\
\text { de } 2015\end{array}$ & 60 min & Jordi Évole \\
\hline
\end{tabular}

Fuente: elaboración propia.

\section{Resultados}

Antes de proceder a la exposición de los resultados, conviene contextualizar brevemente el seguimiento que recibieron los debates televisados objeto de este análisis. Tres de los cuatro debates elegidos para el análisis 
concitaron, de hecho, una gran atención y cosecharon muy buenas cifras de audiencia. ${ }^{7}$ Veamos, en orden descendente, cuáles fueron los debates que más captaron la atención del público. El cara a cara entre el socialista Pedro Sánchez y el presidente popular Mariano Rajoy fue el más seguido de todos los que se pudieron ver durante la campaña electoral. En concreto, el conjunto de espectadores sumó 48.6 \% del share, siendo La Sexta, con $14.6 \%$ de la audiencia, la cadena más sintonizada. El debate a cuatro organizado por Atresmedia, retransmitido simultáneamente por La Sexta y Antena 3, fue líder de audiencia, con más de nueve millones de espectadores y un share de $48.2 \%$; con un pico de $51.3 \%$ durante el llamado minuto de oro. La confrontación entre Albert Rivera y Pablo Iglesias en Salvados, aunque emitida fuera del periodo de la campaña electoral, se convirtió en el programa más visto de la noche del 18 de octubre, con más de cinco millones de espectadores y $25.2 \%$ de cuota de pantalla. Finalmente, el debate a 9, organizado por la cadena pública TVE, solo logró $11 \%$ de share, muy por debajo de los otros debates televisados.

A continuación, se exponen los resultados del análisis temático agrupados en los grandes bloques mencionados (policy issues, political issues, campaign issues o personal issues). De esta forma, podemos observar qué tipo de temas predominaron en las intervenciones de cada uno de los actores políticos en cada uno de los encuentros televisivos seleccionados. Se ha optado por agrupar los temas en estos bloques con el fin de promover la claridad expositiva, puesto que exponer de forma detallada la presencia de setenta categorías temáticas oscurecería la exposición y obstaculizaría la interpretación, pero en algunas ocasiones se detalla la prominencia de ciertos temas específicos, que fueron particularmente destacados.

La tabla 2 contiene los resultados relativos al debate entre Albert Rivera y Pablo Iglesias en Salvados, la tabla 3 aquellos relativos al cara a cara organizado por la Academia de Televisión entre Mariano Rajoy y Pedro Sánchez, la tabla 4 incluye los datos referidos al debate a cuatro organizado por Atresmedia y, finalmente, la tabla 5 recoge los resultados relativos

7 Los datos de audiencia mencionados en este apartado han sido consultados en la base de datos oficial de Kantar Media, http://www.kantarmedia.com/es 
al debate a 9 que organizó la cadena de televisión pública Televisión Española. Todos los datos que se presentan están expresados en porcentajes de columna, de forma que cada una se corresponde con un candidato.

Tabla 2. Los temas del debate entre Albert Rivera (Ciudadanos) y Pablo Iglesias (Podemos) en el programa de La Sexta Salvados

\begin{tabular}{|c|c|c|}
\hline & Pablo Iglesias (Podemos) (\%) & Albert Rivera (Ciudadanos) (\%) \\
\hline Policy & 85.7 & 82.4 \\
\hline Political & 14.3 & 5.9 \\
\hline Campaign & 0 & 11.8 \\
\hline Personal & 0 & 0 \\
\hline
\end{tabular}

Fuente: elaboración propia.

Tabla 3. Los temas del debate entre Pedro Sánchez (PSOE) y Mariano Rajoy (PP) en el encuentro organizado por la Academia de la Televisión

\begin{tabular}{|c|c|c|}
\hline & Mariano Rajoy (PP) (\%) & Pedro Sánchez (PSOE) (\%) \\
\hline Policy & 92.3 & 91.9 \\
\hline Political & 7.7 & 5.9 \\
\hline Campaign & 0 & 0 \\
\hline Personal & 0 & 2.7 \\
\hline
\end{tabular}

Fuente: elaboración propia.

Tabla 4. Los temas del debate entre Pedro Sánchez (PSOE), Pablo Iglesias (Podemos), Albert Rivera (Ciudadanos) y Soraya Sáenz de Santamaría (PP) en el debate organizado por Atresmedia

\begin{tabular}{|c|c|c|c|c|}
\hline & $\begin{array}{c}\text { Sánchez (PSOE) } \\
(\%)\end{array}$ & $\begin{array}{c}\text { Iglesias (Podemos) } \\
(\%)\end{array}$ & $\begin{array}{c}\text { Rivera (Ciudadanos) } \\
(\%)\end{array}$ & $\begin{array}{c}\text { Soraya Sáenz de } \\
\text { Santamaría (PP) (\%) }\end{array}$ \\
\hline Policy & 71.0 & 62.5 & 71.0 & 63.0 \\
\hline Political & 19.4 & 18.8 & 16.1 & 7.4 \\
\hline Campaign & 0 & 0 & 0 & 0 \\
\hline Personal & 0 & 0 & 0 & 7.4 \\
\hline Otros & 9.6 & 18.7 & 12.9 & 22.2 \\
\hline
\end{tabular}

Fuente: elaboración propia. 


\section{Tabla 5. Debate entre Pablo Casado (PP), Antonio Hernando}

(PSOE), Miquel Puig (DiLl), Montserrat Surroca (Unió), Alberto Garzón (IU), Andrés Herzog (UPyD), Aitor Esteban (PNV), Ínigo Errejón (Podemos) y Marta Rivera (Ciudadanos) en el debate organizado por TVE*

\begin{tabular}{|c|c|c|c|c|c|c|c|c|c|}
\hline & $\begin{array}{c}\text { PP } \\
(\%)\end{array}$ & $\begin{array}{c}\text { PSOE } \\
(\%)\end{array}$ & DiLl (\%) & $\begin{array}{c}\text { Unió } \\
(\%)\end{array}$ & $\begin{array}{c}\text { IU } \\
(\%)\end{array}$ & $\begin{array}{c}\text { UPyD } \\
(\%)\end{array}$ & $\begin{array}{c}\text { PNV } \\
(\%)\end{array}$ & $\begin{array}{c}\text { P's } \\
(\%)\end{array}$ & $\begin{array}{c}\text { Ciudadanos } \\
(\%)\end{array}$ \\
\hline Policy & 94.7 & 94.7 & 91.7 & 88.9 & 94.1 & 95.0 & 93.8 & 88.2 & 96.0 \\
\hline Political & 0 & 0 & 0 & 0 & 0 & 0 & 0 & 0 & 0 \\
\hline Campaign & 0 & 0 & 0 & 0 & 0 & 0 & 0 & 0 & 0 \\
\hline Personal & 0 & 0 & 0 & 0 & 0 & 0 & 0 & 0 & 0 \\
\hline Otros & 5.3 & 5.3 & 8.3 & 11.1 & 5.9 & 5 & 6.2 & 11.8 & 4 \\
\hline
\end{tabular}

*Para confeccionar esta tabla hemos optado por utilizar las siglas del partido, con el fin de optimizar el espacio en las columnas y poder observar la comparación completa.

Fuente: elaboración propia.

Resulta indiscutible que las cuestiones de política sectorial, los llamados policy issues según la academia norteamericana, predominan con absoluta claridad en todos los debates analizados. En efecto, las cuestiones relacionadas con aspectos específicos de política pública son, sin duda, aquellas que dominan ampliamente el discurso de todos los candidatos políticos, y lo hacen, además, en todos los encuentros de debate analizados. Por su parte, las grandes referencias a cuestiones políticas en sentido general, es decir, los political issues, que incluyen reflexiones ideológicas y grandes planteamientos programáticos, aparecen en un segundo plano, mientras que las referencias a la propia campaña electoral o a cuestiones de tipo personal son anecdóticas o inapreciables. Este predominio de la política sectorial viene hasta cierto punto condicionado por la propia dinámica de los debates, donde se plantea a los candidatos cuestiones específicas de política pública, es decir, normalmente adscritas a un área concreta y donde se espera que los candidatos expongan sus propuestas específicas con suficiente tiempo y detenimiento. En otras palabras, la dinámica de los debates induce hasta cierto punto esta preferencia por centrar el discurso en diversas cuestiones de política pública. 
Es llamativo, en este sentido, que el contenido del discurso político en los debates electorales esté mucho más volcado hacia la política sectorial que el que llega a la ciudadanía tras la intermediación mediática, normalmente mucho más interesada en otras cuestiones estratégicas, como la política de pactos, los sondeos o los eventos de campaña (Berganza, De Miguel y Chaparro, 2011). En este sentido, existe abundante producción científica en torno a la evolución de la cobertura mediática de las campañas electorales de un enfoque centrado en las cuestiones programáticas a un tratamiento que privilegia la competición política y la estrategia electoral de los actores implicados (Cappella y Jamieson, 1997; Schmuck, Heiss, Matthes, Engesser y Esser, 2016).

Conviene detallar, no obstante, qué cuestiones de política sectorial son las más intensamente destacadas por los candidatos. Si bien agrupamos inicialmente dentro de esta categoría un gran abanico de temas (46 de los 70 posibles temas que diferenciamos en total), en los debates solo aparecieron un número significativamente más reducido de asuntos, en los que los candidatos concentran su atención; por ejemplo, en el debate de Salvados, tres fueron las cuestiones que aparecieron con mayor frecuencia en el discurso de Pablo Iglesias: los impuestos $(21.4 \%$ del total), las pensiones (14.3\%) y las empresas españolas (14.3\%), concebidas tanto como motor de la economía como, sobre todo, en términos críticos sobre su parcialidad ideológica.

Esta particular presencia de las empresas se explica, al menos parcialmente, por las referencias en el debate de La Sexta de unas declaraciones del presidente del BBVA, Francisco González, crítico con Podemos. González advirtió de los riesgos que supondría para la economía española la aplicación de las medidas propuestas por Pablo Iglesias; por contraste, alababa a Albert Rivera como posible presidenciable. El moderador del programa, Jordi Évole, reprodujo las declaraciones para que ambos dirigentes políticos opinasen al respecto.

Por su parte, en los mensajes de Albert Rivera también destacaron los impuestos (11.8\%), pero la atención también se centró en temas como la lucha contra el terrorismo (11.8 \%) y el independentismo catalán (tam- 
bién $11.8 \%$ ), cuestiones ambas que han definido a Ciudadanos como partido político desde su fundación en Cataluña en 2007 en cuanto reacción a la hegemonía del nacionalismo catalán, y que han permanecido como señas de identidad, al menos hasta cierto punto, después de que el partido se transformara en una formación de alcance nacional.

Frente a estas diferencias entre las agendas temáticas resaltadas por los candidatos de los nuevos partidos en el formato de La Sexta, el cara a cara organizado por la Academia de la Televisión entre Pedro Sánchez y Mariano Rajoy mostró una distribución temática más similar entre candidatos. Posiblemente como resultado, al menos en parte, de un formato más tradicional y encorsetado, que dejaba menos margen a los candidatos para desviarse en sus intervenciones de las cuestiones planteadas por el moderador en cada bloque temático. Así, los tres temas más destacados por Pedro Sánchez fueron los mismos que sobresalieron en el discurso de Mariano Rajoy: la corrupción (18.9 \% Sánchez y 12.9 \% Rajoy), el paro (13.5 \% Sánchez y $15.4 \%$ Rajoy) y, por último, la economía (8.1 \% Sánchez y $17.9 \%$ Rajoy). Sin embargo, existen diferencias entre candidatos, de forma que cada uno de ellos incidió en aquellos asuntos que les resultaban electoralmente más ventajosos: la gestión de la economía en el caso de Rajoy, cuya estrategia de campaña estaba basada en la idea de haber iniciado la recuperación económica, y las denuncias de los casos de corrupción en el de Pedro Sánchez, cuya campaña estaba basada en denunciar la crisis multiorgánica del país y en incidir en los casos de corrupción vinculados con el PP (Valera et al., 2017).

En el debate de Atresmedia, hubo tres temas que centraron la mayor parte del interés de los candidatos: el desempleo (7.4\%), la corrupción $(7.4 \%)$ y los recortes $(5.8 \%)$.

No obstante, cada candidato trató de forma diferente estas tres cuestiones, bien a través de intervenciones directas, bien por ausencia de respuestas a las alusiones que hacía el resto de candidatos. Conviene destacar también la presencia singular de algunos temas en las intervenciones de un único candidato. Es el caso de la organización territorial del Estado, al que Albert Rivera dedicó $6.5 \%$ de sus intervenciones, frente a porcentajes insignificantes en el caso de los otros candidatos; o el del independentismo 
catalán, al que Soraya Sáenz de Santamaría también prestó más atención (7.4\%) que el resto de los candidatos (3.15\%).

En el debate más plural de TVE, en el que participaban nueve fuerzas políticas, los temas relativos a la corrupción (10.4\%) y el desempleo (16.2\%) fueron los que acapararon el mayor interés de los representantes políticos. Fue, no obstante, la cuestión de la organización territorial del Estado la que ocupó el tercer lugar, con 9.7 \% de las intervenciones totales. Sin duda, la presencia de los candidatos nacionalistas espoleó este porcentaje. Aitor Esteban (Partido Nacionalista Vasco) monopolizó 31.3 \% de las intervenciones al respecto, por $11.1 \%$ de Miquel Puig (Democracia i Llibertat) y $8.3 \%$ de Montserrat Surroca (Unió). En el lado de los constitucionalistas, Pablo Casado (PP) dedicó $10.5 \%$ del recuento observado y Marta Rivera (Ciudadanos) $12.0 \%$ de las ocasiones.

\section{Conclusiones}

Los debates electorales revisten una relevancia singular, pues suponen una escenificación y confrontación directa del debate político que se produce entre distintos candidatos durante la campaña electoral. En ellos, los actores políticos tratan de influir en la agenda mediática y, en última instancia, en la percepción que tiene el electorado de los problemas que afectan a la sociedad. Asimismo, constituyen auténticos acontecimientos mediáticos, tanto en lo que se refiere a las audiencias que concitan como respecto del volumen de información que generan (noticias, comentarios, análisis, etc.).

Este trabajo de investigación se ha interrogado sobre el tipo de cuestiones que tienden a prevalecer en el discurso de los actores políticos durante los debates electorales con motivo de la campaña electoral española de 2015, con el fin último de establecer hasta qué punto estos espacios conservan su función como foros para el debate de política sectorial y la discusión de políticas públicas, frente a la tendencia de la gran mayoría de los espacios y las situaciones inscritos en la comunicación política hacia la mediatización (Blumler y Kavanagh, 1999; Couldry y Hepp, 2013; Schmuck et al., 2016). No obstante, conviene poner de manifiesto que el análisis aquí presentado se circunscribe a un estudio de caso específico, relativo a las 
elecciones generales que se produjeron en España en diciembre de 2015, por lo cual los resultados no pueden extrapolarse a otros contextos políticos. Las futuras investigaciones deberán, en este sentido, profundizar en esta línea de investigación mediante aproximaciones comparadas.

Mediante un análisis de contenido sistemático, hemos establecido claramente que los debates electorales permiten a los candidatos, al menos en el caso español, encauzar sus prioridades temáticas y de política pública y contrastarlas con sus rivales ante el público, con una intermediación (o mediatización) significativamente menor que la que habitualmente ejercen los medios de comunicación. En el caso de los debates, el medio (en este caso, la televisión) tiene un papel más secundario que el que ejerce habitualmente en la elaboración de productivos informativos. Naturalmente, el medio puede condicionar el debate a través del formato (habitualmente consensuado con los partidos) y con las preguntas (a veces, también consensuadas), pero el protagonismo corresponde enteramente a los candidatos, y ello permite que estos espacios conserven su función de alojar un debate detallado de cuestiones relativas a diferentes ámbitos de política sectorial.

Los resultados de esta investigación subrayan, por tanto, que los debates electorales constituyen un espacio relativamente poco mediatizado en el contexto general de mediatización de la política que se produce en las democracias contemporáneas. Y además, lo hacen con motivo de los cuatro encuentros electorales analizados, que exhiben una variedad de formatos y participantes políticos. Naturalmente, los candidatos tendieron a destacar aquellos temas que les resultaban electoralmente más ventajosos, y que constituyen las líneas de fuerza de su apuesta de intervención sobre la realidad, como la oposición al independentismo catalán de Albert Rivera (Ciudadanos) o el énfasis de Pedro Sánchez (PSOE) en denunciar la corrupción del PP. Pero, en todos los casos, los debates estuvieron claramente centrados en el análisis de cuestiones de política sectorial, y prestaron escasa atención a otros temas generalmente asociados con el proceso de mediatización, como aquellos referidos al desarrollo de la campaña o con la personalidad de los líderes. En definitiva, podemos afirmar que los debates electorales constituyeron un reducto frente a la mediatización de la comunicación política contemporánea. 
Cuestión diferente, por supuesto, es toda la actividad que rodeó los debates electorales, es decir, los diversos programas y formatos mediáticos dedicados a comentar los debates antes y después de que se produjeran, la actividad generada a causa de los encuentros en las redes sociales, las encuestas (también elaboradas desde los medios) destinadas a dibujar un ganador del debate, etc. En casi todos estos casos, los medios recuperaron el protagonismo en la producción de los mensajes, lo cual previsiblemente alteró la naturaleza de estos y desplazó la atención de las cuestiones que fueron realmente debatidas en estos encuentros hacia otro tipo de cuestiones, como los efectos de los debates sobre el público (qué candidato resultó vencedor a los ojos del público, qué influencia sobre el voto podrían tener los debates, etc.). Un caso extremo de esta disociación entre las cuestiones que dominaron el debate en sí y la perspectiva sobre el debate que aportaron los medios (la más determinante) se produjo con motivo del encuentro entre los líderes de los nuevos partidos en el caso de Salvados. Martín, Reguero y Velasco (2015) destacan que los medios de comunicación no trataron apenas los contenidos de este debate, sino que se centraron exclusivamente en lo novedoso del formato y en el papel de la televisión como vehículo para focalizar la atención de los ciudadanos en materia política. Los futuros estudios deberán ocuparse de analizar el papel de los medios en lo que podríamos llamar el "debate del debate", es decir, de estudiar qué tipo de cuestiones prevalecieron en estos espacios, con el fin de obtener una visión de conjunto de su incidencia en el proceso de la campaña electoral.

\section{Referencias}

Barreiro, X., Pereira, L. M. y García, G. (2015). Los efectos sobre el voto de la campaña electoral en las elecciones europeas de 2014 en España. Revista Española de Ciencia Política, 39, 67-93.

Bennett, L. e Iyengar, S. (2008). A new era of minimal effects? The changing foundations of political communication. Journal of Communication, 58, 707-731. 
Berganza R., De Miguel, P. y Chaparro, M. A. (2011). La prensa gratuita ante las elecciones generales de España del 9 de marzo de 2008: cobertura, politización y frames en contraposición a la prensa de pago. Comunicación y Sociedad, 24(1), 35-61.

Blumler, J. y Gurevitch, M. (1995). The crisis of public communication. Londres: Routledge.

Blumler, J. y Kavanagh, D. (1999). The third age of political communication: Influences and Features. Political Communication, 16, 206-230.

Cappella, J. N. y Jamieson, K. H. (1997). News frames, political cynicism, and media cynicism. The annals of the American Academy of Political and Social Science, 546, 71-84.

Ceron, A. y Splendore, S. (2016). From contents to comments: Social TV and perceived pluralism in political talk shows. New Media \& Society, 1-17. DOI: 10.1177/1461444816668187

Chadwick, A. (2013). The hybrid media system: Politics and power. Nueva York: Oxford University Press.

Couldry, N. y Hepp, A. (eds.) (2013). Conceptualizing mediatization. Communication Theory, 23(3), 191-202.

García, J. (2015). La cobertura mediática de los debates electorales en España. Revista Española de Ciencia Política, 38, 135-161.

García, Ó. (2011). Debates electorales en televisión: una aproximación preliminar a sus efectos inmediatos. Revista Española de Ciencia Política, 25, 81-96.

Goldman, S. y Mutz, D. (2011). The friendly media phenomenon: A crossnational analysis of cross-cutting exposure. Political Communication, 28, 42-66. 
Humanes, M.L. (2014). Exposición selectiva y partidismo de las audiencias en España: el consumo de información política durante las campañas electorales de 2008 y 2011. Palabra Clave, 17(3), 773-802.

Iyengar, S. y Kinder, D. (2010). News that matters: Television and american opinion, updated edition. Chicago: Chicago University Press.

Lago, I. y Martínez, F. (2013). Apuntes sobre el estudio del comportamiento electoral en España. Revista de Estudios Políticos, 161, 69-91.

López, G., Gámir, J. V., García, F. J., Llorca, G., Cano, L. y González, J. L. (2015). El debate sobre Europa en Twitter: discursos y estrategias de los candidatos de las elecciones al Parlamento Europeo de 2014 en España. Revista de Estudios Políticos, 170, 213-246.

López-García, G. y Valera-Ordaz, L. (ed.) (2017). Pantallas electorales: el discurso de partidos, medios y ciudadanos en la campaña de 2015. Barcelona: UOC.

Martín, V., Reguero, I. y Velasco, A. (2015). El debate del debate: Salvados y el politainment como generador de contenidos en la prensa tradicional. En C. Mateos y J. Herrero (coords.), La pantalla insomne (pp. 1449-1467). San Cristóbal de La Laguna: Universidad de La Laguna.

Mazzoleni, G. (2010). La comunicación política. Madrid: Alianza.

McCombs, M. y Shaw, D. (1972). The agenda setting function of the mass media. Public Opinion Quarterly, 36(2), 176-187.

Patterson, T. E. (1980). The mass media election: How americans choose their president. Nueva York: Praeger.

Rodríguez, R. y Bouza, F. (2008). Cambios en las agendas y cambios en la comunicación política. En M. J. Canel Crespo y M. García Gu- 
rrionero (coords.), Estudios de comunicación política (pp. 45-64). Madrid: Asociación Comunicación Política.

Rodríguez-Virgili, J., Jandura, O. y Rebolledo de la Calle, M. (2014). La personalización de la política en la cobertura mediática: una comparación de las campañas electorales en España y América. Trípodos, 34, 61-79.

Schmuck, D., Heiss, R., Matthes, J., Engesser, S. y Esser, F. (2016). Antecedents of strategic game framing in political news coverage. Journalism, 1-19.

Schrott, P. (1990). Electoral consequences of 'winning' televised campaign debates. Public Opinion Quarterly, 54(4), 567-585.

Schulz, W. (2004). Reconstructing mediatization as an analytical concept. European Journal of Communication, 19, 87-101.

Strömbäck, J. (2008). Four phases of mediatization: An analysis of the mediatization of politics. International Journal of Press/Politics, 13, 228-246.

Vaccari, C., Chadwick, A. y O'Loughlin, B. (2015). Dual screening the political: Media events, social media, and citizen engagement. Journal of Communication, 65, 1041-1061.

Valera-Ordaz, L., Carratalá, A. y Palau, D. (2017). La batalla de los partidos por la definición de la realidad: los marcos partidistas durante las elecciones generales 2015. En G. López-García y L. Valera-Ordaz (eds.), Pantallas electorales: el discurso de partidos, medios y ciudadanos en la campaña de 2015 (pp. 59-78). Barcelona: UOC.

Vergeer, M. y Franses, P. H. (2016). Live audience responses to live televised election debates: Time series analysis of issue salience and party salience on audience behavior. Information, Communication \& Society, 19(10), 1390-1410. 
Zaller, J. (1992). The nature and origins of mass opinion. Nueva York: Cambridge University Press.

\section{Anexo 1. Temas de debate}

\section{A. Policy issues}

1) Empleo/desempleo

2) Impuestos

3) Recortes económicos y sociales

4) Deuda pública

5) Vivienda

6) Balanzas fiscales

7) Desigualdades

8) Empresas

9) Turismo

10) Economía (otras cuestiones)

11) Educación

12) Sanidad

13) Seguridad ciudadana

14) Familia

15) Inmigración

16) Pensiones

17) Organización territorial del Estado

18) Nacionalismo

19) Independentismo catalán

20) $15 \mathrm{M}$

21) Emigrantes, españoles en el exterior

22) Infraestructuras

23) Manipulación informativa, medios públicos

24) Manipulación informativa, medios privados

25) Política internacional

26) Cultura

27) Canon digital, derechos de autor

28) Sociedad de la información/nuevas tecnologías

29) Políticas de igualdad/derechos sociales 
30) Ley del aborto

31) Utilización electoralista de fondos públicos

32) Agricultura

33) Unión Europea

34) Problemas urbanísticos

35) Industria

36) Medio ambiente

37) Memoria Histórica

38) Justicia

39) Iglesia, relaciones con la iglesia/catolicismo

40) Fiestas, tradiciones

41) Corrupción

42) Grandes proyectos

43) Deportes

44) Violencia de género

45) Terrorismo

46) Puertas giratorias

47) Policy issues (genérico)

\section{B) Political issues}

48) Sondeos

49) Coaliciones de partidos

50) Estimación de resultados

51) Programas electorales

52) Participación vs. Abstención

53) Participación ciudadana

54) Debates electorales

55) Crispación

56) Relación con actores sociales

57) Política interna de partido

58) Resultados electorales

59) Regeneración democrática

60) Voto rogado (voto por correo desde el extranjero)

61) Political issues (genérico) 


\section{C) Campaign issues}

62) Eventos de campaña

63) Organización de campaña

64) Estrategias de campaña

65) Anécdotas, aspectos no políticos de la campaña (cuestiones estéticas, frivolidades, etc.).

66) Campaign issues (genérico)

\section{D) Personal issues}

67) No se aplica/temas ajenos a la campaña electoral

68) Candidatos (personalidad, cualidades)

69) Personal issues

70) Otros 